\title{
NO EXISTENCIA DE FUNCIONES CONTINUAS ENTRE CONTINUOS HEREDITARIAMENTE DESCOMPONIBLES E INDESCOMPONIBLES
}

\author{
${\text { William } \text { Olano }^{1}, \text { Adrian Aliaga }}^{2}$, Marco Rubio ${ }^{3}$ \\ (Recibido: 15/10/2015 - Aceptado: 30/11/2015)
}

Resumen: En el presente artículo detallaremos una demostración de la proposición: no existencia de funciones continuas entre continuos hereditariamente descomponibles e indescomponibles.

Palabras clave: Continuos, continuos hereditariamente descomponibles y continuos indescomponible.

\section{NO EXISTENCE OF CONTINUOUS FUNCTIONS BETWEEN CONTINUOUS HEREDITARILY DECOMPOSABLE AND INDECOMPOSABLE}

\begin{abstract}
In this paper will detail a demonstration of the proposition : absence of functions between continuous decomposable and indecomposable hereditarily.

Keywords: Continuous, on going and continuous indecomposable hereditarily decomposable.
\end{abstract}

\section{Introducción}

El presente trabajo pertenece a la rama de la topología conocida como Teoría de los Continuos. Dicha temática trata del estudio de las propiedades topológicas de los espacios que son métricos, no vacíos, compactos y conexos. A un espacio topológico con las propiedades antes mencionadas se le llama continuo. Mediante la teoría de continuos detallaremos la demostración del siguiente teorema: ¿exitirán funciones continuas entre continuos hereditariamente descomponibles e indescomponibles? La respuesta es no ([4]).

Este último resultado abre una línea de trabajo en el campo de la topología y más concretamente en la de los encajes ordenados en hiperespacios de continuos ([1]).

\section{Prelimirares}

Definición 2.1 Un continuo es un espacio métrico, conexo, compacto y no vacío.

Definición 2.2 Sean $X$ un continuo y $A$ un subconjunto de $X$. Se dice que $A$ es un subcontinuo de $X$, si $A$ es un continuo.

Definición 2.3 Un continuo es no degenerado si contiene más de un punto. En caso contrario, diremos que el continuo es degenerado.

\footnotetext{
${ }^{1}$ UNMSM, Facultad de Ciencias Matemáticas, e-mail: wcolano@hotmail

${ }^{2}$ UNMSM, Facultad de Ciencias Matemáticas, e-mail: agaliagall@hotmail.com

${ }^{3}$ UNMSM, Facultad de Ciencias Matemáticas, e-mail: antoniorubiog@gmail.com
} 
Ejemplos de continuos son: El intervalo unitario, el círculo, el triodo, la figura ocho, cualquier gráfica finita, el toro, la cerradura de la gráfica de la función sen $\frac{1}{x}$, el círculo de Varsovia, el cono del conjunto de Cantor y muchísimos más, que usted podrá encontrar en artículos escritos sobre hiperespacios de continuos ([1]).

Definición 2.4 Un continuo $X$ es descomponible si $X=A \cup B$, donde $A$ y $B$ son subcontinuos propios de $X$. Diremos que un continuo $X$ es indescomponible si no es descomponible.

Es un hecho conocido que el continuo de Igram, el arco iris de Knaster y el selenoide diadico son continuos indescomponibles.

Definición 2.5 Sea $X$ un continuo. Decimos que $X$ es hereditariamente descomponible si cada continuo $Y \subset X$ con más de un punto es descomponible.

Definición 2.6 Una colección de subconjuntos $\mathcal{F}$ de un espacio $X$ es una cadena en $X$ si para cualesquiera elementos $A$ y $B$ en $\mathcal{F}$ se tiene que $A \subset B$ o $B \subset A$.

Definición 2.7 Una colección $\mathcal{C}$ de subconjunto de $X$ se dice que tiene la propiedad de la intersección finita si cada subcoleción finita $\left\{C_{1}, \ldots, C_{n}\right\}$ de $\mathcal{C}$ se tiene la intersección no vacía, es decir, $C_{1} \cap \ldots \cap C_{n}$ es no vacía.

Definición 2.8 Dado un conjunto $A$, una relación $\leq$ sobre $A$ se denomina orden parcial estricto sobre A si tiene las siguientes propiedades:

a) (No-reflexiva) la relación $a \leq a$, nunca se cumple.

b) (Transitividad) si $a \leq b$ y $b \leq c$, entonces $a \leq c$.

Definición 2.9 Sea $\alpha$ elemento de un conjunto parcialmente ordenado $\Gamma$, por una relación $\leq$. Decimos que $\alpha$ es un elemento minimal en $\Gamma$ si, para todo $\gamma \in \Gamma$ tal que $\gamma \leq \alpha$, se tiene que $\gamma=\alpha$.

Notación.- Sea $\mathcal{C}$ una colección de subconjuntos de $X$. La intersección de los elementos de $\mathcal{C}$, la denotaremos por $\bigcap \mathcal{C}$. Es decir, $\bigcap_{C \in \mathcal{C}} C$.

Teorema 2.10 Si $\mathcal{F}$ es una cadena de conjuntos cerrados en un espacio compacto $X$. Sea $U$ un conjunto abierto de $X$ tal que $\bigcap \mathcal{F} \subset U$, entonces existe un elemento $A$ en $\mathcal{F}$ tal que $A \subset U$.

\section{Demostración}

Sea $X$ y $\mathcal{F}$ como se indican. Supongamos que, para todo $A \in \mathcal{F}, A$ no contenido propiamente en $U$. Denotemos $\mathcal{A}=\{A-U \mid A \in \mathcal{F}\}$. Se tiene que $\mathcal{A}$ es una colección de subconjuntos cerrados y no vacíos de $X$. Veamos que la colección $\mathcal{A}$ tiene la propiedad de la intersección finita: como $\mathcal{F}$ es una cadena, para cada subcolección finita, $A_{1}, \ldots, A_{n}$, de elementos de $\mathcal{F}$, existe $i \in\{1, \ldots, n\}$ tal que $A_{i} \subset A_{j}$ para todo $j \in\{1, \ldots, n\}$, luego, $\bigcap_{j=1}^{n} A_{j}-U=A_{i}-U \neq \emptyset$.

Ahora, como $X$ es compacto, se sigue que $\bigcap \mathcal{A} \neq \emptyset,([3], 5.9 ; 17)$. Luego, puesto que $\bigcap \mathcal{A}=(\bigcap \mathcal{F})-U$, se obtiene que $\bigcap \mathcal{F}$ no esta contenido propiamente en $U$. Así, el teorema está demostrado.

Teorema 2.11 Si $\mathcal{F}$ es una cadena de subconjuntos cerrados, conexos y no vacios, de un espacio de Hausdorff, compacto y conexo $X$, entonces $\bigcap \mathcal{F}$ es un conjunto compacto, conexo y no vacío en $X$. 


\section{Demostración}

Como la intersección de conjuntos cerrados es un conjunto cerrado, se tiene que $\bigcap \mathcal{F}$ es un conjunto cerrado y, es un conjunto compacto en $X$. Por otra parte, notemos que $\mathcal{F}$ tiene la propiedad de la intersección finita (ya que es una cadena de conjuntos no vacíos), luego, por la compacidad de $X$, se obtiene que $\bigcap \mathcal{F} \neq \emptyset$, ([3],5.9;17). Así, resta mostrar que $\bigcap_{\mathcal{F}}$ es conexo.

Supongamos que $\bigcap \mathcal{F}=A \cup B$, donde $A$ y $B$ son conjuntos cerrados y disjuntos. Probaremos que $A=\emptyset$ о $B=\emptyset$. Como $X$ es de Hausdorff y compacto, se tiene que $X$ es un espacio normal, así podemos considerar abiertos $U$ y $V$, en $X$ tales que $A \subset U, B \subset V$ y $U \cap V=\emptyset$. Se tiene que $\bigcap \mathcal{F} \subset U \cup V$ y $U \cup V$ es un conjunto abierto en $X$. Luego, por el Teorema 2.10, existe un elemento $F \in \mathcal{F}$ tal que $F \subset U \cup V$. Como $F$ es conexo y $U$ y $V$ son abiertos disjuntos, se tiene que $F \subset U$ o $F \subset V$. Sin perder generalidad, suponemos que $F \subset U$. Ahora, puesto que $\bigcap \mathcal{F} \subset F$, se tiene que $A \cup B \subset F$ y, en consecuencia, $B \subset U$. Así $B \subset U \cap V=\emptyset$, por lo cual $B=\emptyset$. Esto prueba que $\bigcap \mathcal{F}$ es conexo.

\section{Resultado principal}

Teorema 3.1 (Teorema de reducción de Brouwer)

Sean $X$ un espacio métrico con una base numerable y $\mathcal{C}$ una colección de subconjuntos cerrados de $X$, ordenada parcialmente por la inclusión de conjuntos. Si para toda sucesión decreciente en $\mathcal{C},\left\{C_{n}\right\}_{n \in \mathbb{N}}$, se tiene que $\bigcap_{n \in \mathbb{N}} C_{n} \in \mathcal{C}$, entonces existe un elemento minimal en $\mathcal{C}$.

\section{Demostración}

Fijemos una base numerable para $X$, digamos $\left\{B_{n} \mid n \in \mathbb{N}\right\}$ y un elemento $C_{0} \in \mathcal{C}$. Denotemos

$$
\mathcal{C}_{0}=\left\{C \in \mathcal{C} \mid C \subset C_{0} \cap\left(X-B_{1}\right)\right\} .
$$

Si la colección $\mathcal{C}_{0}$ es no vacía, fijamos un elemento $C_{1} \in \mathcal{C}_{0}$ y, si la colección $\mathcal{C}_{0}$ es vacía, definimos $C_{1}=C_{0}$. Ahora supongamos que se han determinado $n$ elementos de $\mathcal{C}$, digamos $C_{1}, C_{2}, \ldots, C_{n}$ tales que $C_{i} \subset C_{i-1} \subset C_{0}$ y, si $C_{i}$ está contenido propiamente en $C_{i-1}$, entonces $C_{i} \cap B_{i}=\emptyset$, para cada $i \in\{1, \ldots, n\}$. Denotemos

$$
\mathcal{C}_{n}=\left\{C \in \mathcal{C} \mid C \subset C_{n} \cap\left(X-B_{n+1}\right)\right\} .
$$

Si la colección $\mathcal{C}_{n}$ es no vacía, fijamos un elemento $C_{n+1} \in \mathcal{C}_{n}$ y, si la colección $\mathcal{C}_{n}$ es vacía, definimos $C_{n+1}=C_{n}$.

De este modo, inductivamente, hemos determinado una sucesión decreciente, $\left\{C_{n}\right\}_{n \in \mathbb{N}}$, de elementos de $\mathcal{C}$ con la propiedad adicional de que, para cada $n \in \mathbb{N}$, si $C_{n+1}$ está contenido propiamente en $C_{n}$, entonces $C_{n+1} \cap B_{n+1}=\emptyset$.

Denotemos $E=\bigcap_{n \in \mathbb{N}} C_{n}$. Por hipótesis se tiene que $E \in \mathcal{C}$.

Vamos a probar que $E$ es un elemento minimal en $\mathcal{C}$.

Para esto supongamos lo contrario y fijemos $D \subset E$ con $D \neq E$ tal que $D \in \mathcal{C}$. Fijemos un punto $x \in E-D$. Como $D$ es cerrado, existe $k \in \mathbb{N}$ tal que $x \in B_{k} \subset(X-D)$. Notemos que

$$
B_{k} \cap E \neq \emptyset, B_{k} \cap D=\emptyset \quad \text { y } D \subset E \subset C_{k-1} .
$$

Se sigue que $D \subset C_{k-1} \cap\left(X-B_{k}\right)$, así, $C_{k-1} \neq \emptyset$. Luego, $C_{k}$ está determinado de modo que $C_{k} \in \mathcal{C}$ y $C_{k} \subset C_{k-1} \cap\left(X-B_{k}\right)$. Dado que $E \subset C_{k} \subset\left(X-B_{k}\right)$, se obtiene $E \cap B_{k}=\emptyset$ lo cual es una contradicción. Esta contradicción demuestra que $E$ es un elemento minimal en $\mathcal{C}$.

Para probar la "no existencia de funciones continuas entre continuos hereditariamente descomponibles e indescomponibles" utilizaremos el Teorema de reducción de Brouwer. 
Teorema 3.2 Si $X$ es un continuo hereditariamente descomponible e $Y$ es un continuo indescomponible, entonces no existen funciones continuas de $X$ sobre $Y$.

\section{Demostración}

Supongamos que exista una función continua y suprayectiva $f: X \longrightarrow Y$. Consideremos el conjunto

$$
\mathcal{Z}=\{A \subset X \mid A \text { es un continuo y } f(A)=Y\},
$$

el cual es no vacío por hipótesis. Si $\mathcal{C} \subset \mathcal{Z}$ es una cadena, sea $C_{0}=\bigcap \mathcal{C}$ que es un continuo (por el Teorema (2.11)). Vamos a probar que $f\left(C_{0}\right)=Y$, para esto, sea $y \in Y$. Para cada $C \in \mathcal{C}$, sabemos que $f^{-1}(y) \cap C$ es compacto no vacío. La familia $\left\{f^{-1}(y) \cap C \mid C \in \mathcal{C}\right\}$ tiene la propiedad de la intersección finita así que su intersección es no vacía, $f^{-1}(y) \cap C_{0} \neq \emptyset$. Por lo tanto, $f\left(C_{0}\right)=Y$.

Por el Teorema de reducción de Brouwer, existe $M \in \mathcal{Z}$ minimal. Notemos que $M$ es indescomponible. De lo contrario, al escribir $M=A \cup B$ con $A, B \subset M$ subcontinuos propios, $Y=f(M)=f(A) \cup f(B)$; por la minimalidad de $M, f(A)$ y $f(B)$ son subcontinuos propios, lo cual implica que $Y$ es descomponible. Esta contradicción completa la prueba.

\section{Conclusión}

Hay muchos artículos escritos sobre hiperespacios de continuos. Sería imposible incluir todos los posibles resultados. Hemos presentado algunos de los más representativos. Esperamos que el lector llegue a la conclusión de que el conjunto potencia de un continuo es dificil, que sus subconjuntos que llamamos hiperespacios de subcontinuos tienen una bonita estructura y que, además, son más aceptables que los continuos en general. 


\section{REFERENCIAS BIBLIOGRÁFICAS}

[1] Andablo, G. (2009). Funciones entre hiperespacios de continuos y relación de orden. Memoria de la XIX Semana Regional de Investigación y Docencia en Matemáticas. Departamento de Matemáticas. Universidad de Sonora, México. Mosaicos Matemáticos No 32: 163-169.

[2] Robles Corbal, Carlos A., Andrade Espinoza, Martha P. (2007). Algunas técnicas para la construcción de continuos indescomponibles. Memoria de la XVII Semana Regional de Investigación y Docencia en Matemáticas. Universidad de Sonora. Departamento de Matemáticas. Mosaicos Matemáticos No. 20; 151-161.

[3] Munkres, James Raymond (1975). Topology, a first course. Prentice-Hall, Inc., New Jersey.

[4] Wlodzimierz Charatonik (2010). Propiedades que se preservan bajo Funciones Confluentes. IV Taller de Continuos e Hiperespacios. Morelia, Michoacan. 